\title{
LA COPIE DU PRÉNOM EN GRANDE SECTION : DIMINUTION DE LA TAILLE DES CARACTÈRES
}

\begin{abstract}
Résumé : L'article pose le problème de la réduction de la taille des lettres, en grande section, lors de l'écriture du prénom. Nous émettons l'hypothèse que le modèle de l'étiquette prénom ainsi que les dimensions du support papier ne sont pas sans conséquence sur les difficultés des élèves, lorsqu'il s'agit pour eux d'écrire entre deux lignes et par là même, de réduire leur production scripturale dès l'entrée au C.P.

Mots-clés : Ecole maternelle, Grande Section, copie du prénom, étiquette modèle, diminution des caractères copiés, réduction du format du support papier, liaison G.S./C.P.
\end{abstract}

L'étiquette prénom est l'outil de référence de la construction identitaire de l'élève à l'école maternelle. Conçue par l'enseignant dès la première scolarisation de l'enfant, elle est présente en différents lieux de l'école: dans la classe tout d'abord (tableau des présents et absents, tableau des responsabilités, cahiers des élèves, bureaux individuellement repérés, casiers de rangement des productions, anniversaires...) mais également dans le couloir, les salles collectives (portemanteaux, casiers des serviettes à la cantine....). L'objectif est prioritairement un objectif de lecture afin de favoriser pour chacun, la reconnaissance visuelle de son prénom puis la reconnaissance d'autres prénoms d'élèves de la classe.

Outre ces étiquettes figées donnant à l'élève des repères, une autre étiquette prénom mobile cette fois, peut servir de support aux activités de lecture et d'écriture. Des enseignants font le choix de présenter le prénom en capitales d'imprimerie (P.S./M.S.) ; d'autres en lettres scriptes minuscules (M.S./G.S.) ; d'autres encore transcrivent les différentes graphies sur la même étiquette (capitales d'imprimerie, lettres scriptes minuscules et cursives) d'autant que les élèves sont plus âgés. Les programmes $2002^{1}$ préconisant le passage de l'écriture bâton à l'écriture cursive, certains maîtres différencient les étiquettes lecture (privilégiant l'usage des lettres scriptes minuscules comme dans les livres) des étiquettes écriture. Ces dernières présentent alors le plus souvent un modèle en capitales d'imprimerie qui fera place, dès que possible, pour chaque enfant, à l'écriture cursive.

\footnotetext{
1 «Qu'apprend-on à l'école maternelle? » Ministère de l'Éducation Nationale. Paris : CNDP, XO Éditions, p. 87.
}

SPIRALE - Revue de Recherches en Éducation - $2005 N^{\circ} 36$ (99-108) 


\section{S. BRIQUET-DUHAZÉ}

A partir d'observations de pratiques pédagogiques récentes et des études sur l'acte d'écrire des années soixante à aujourd'hui, je me suis donc interrogée sur un point précis concernant l'écriture du prénom et la capacité ou non de l'élève à diminuer la taille des caractères afin d'évaluer sa maitrise et de mieux cerner l'origine des difficultés d'écriture sur des formats plus petits que ceux utilisés le plus souvent à l'école maternelle. J'ai mené une expérimentation en deux temps. L'observation des productions des élèves lorsque l'étiquette prénom modèle était présentée dans un petit format, puis lorsque le support lui-même était réduit.

\section{CONSTATS À PROPOS DES MODÈLES D'ÉTIQUETTES PRÉNOMS}

Mon expérience d'institutrice en maternelle pendant treize ans et mes observations des étiquettes prénoms modèles, proposées par les enseignants aux élèves, m'ont permis de constater les faits suivants.

La taille de ces étiquettes, dans un très grand nombre d'écoles maternelles, varie peu. Il s'agit le plus souvent, de fiches cartonnées de format $20 X 12 \mathrm{~cm} . \mathrm{Ce}$ format relève d'un choix particulier concernant la bonne visibilité pour l'élève, du tracé des lettres, de leur sens, des espaces entre elles... Cela induit, de la part de l'enseignant, une modélisation de sa propre police qui varie peu : j'ai pu vérifier qu'en moyenne, la hauteur des lettres modèles, sur l'étiquette prénom, était d'environ $2,5 \mathrm{~cm}$, que l'enseignant écrive lui-même ce modèle ou bien qu'il soit réalisé avec un traitement de texte. C'est aussi la hauteur des lettres utilisée pour les étiquettes de lecture ou pour toute autre conception d'affiches murales par le maître.

Quelle que soit la section considérée (P.S. à G.S.), ce format demeure le même, seul le choix de l'écriture varie (capitale d'imprimerie, cursive...).

D'autre part, j'ai également observé les supports sur lesquels les élèves reproduisaient leur prénom. Il s'agit majoritairement de feuilles de papier machine de format A4 destinées aux travaux écrits de lecture, de mathématiques, de graphisme et même d'écriture. L'emploi de ce support s'explique par la reproduction photocopiée de l'exercice très largement utilisée dans les écoles.

Enfin, j'ai prêté une attention particulière aux caractéristiques développées par les enseignants lorsqu'ils étaient en position de scripteur modèle, devant leurs élèves. Que ce soit pour réaliser des affiches murales ou bien lors de l'écriture au tableau, en grande section, les maîtres usent d'une certaine amplitude du geste d'autant qu'ils réduisent leur vitesse d'écriture afin que les élèves mémorisent mieux le tracé. Lorsqu'ils copient le modèle du prénom, devant l'élève et au dos d'une feuille de travail notamment, les professeurs réduisent également leur vitesse d'exécution, insistent sur les mouvements du poignet, cela participant inévitablement à l'agrandissement des caractères proposés.

Nous pouvons donc considérer, qu'à l'école maternelle, l'élève a sous les yeux des productions scripturales de la part de l'enseignant (affiches murales, étiquettes...) dont le modèle de hauteur des lettres en particulier, n'est pas le reflet de celle que l'enfant utilisera dès l'apprentissage officiel de l'écriture sur des supports 
formels tels que le cahier. En effet, dès l'entrée à l'école élémentaire, l'élève possède des modèles d'écriture de la part du maitre dont la taille des caractères est le modèle communément admis et utilisé tout au long de la scolarité (poésies, exercices divers, leçons, modèles d'écriture...) et de la vie. Les affiches sont également présentes (comme l'alphabet, les règles diverses...) mais à l'école maternelle, on note un agrandissement quasi-systématique des caractères d'autant que les supports formels sont absents. Cette constatation mériterait certainement que l'on s'y attarde mais ne fera pas l'objet de notre questionnement.

Au cours de la grande section, il n'est pas rare que l'enseignant propose un cahier d'écriture aux élèves. Même si ce constat n'est pas unanime, l'observation des productions des élèves montre que pour beaucoup, la diminution des caractères produits est une réelle difficulté. Cette activité d'écriture demande à l'élève une mobilisation conjointe de multiples compétences, outre celle qui fait l'objet de notre étude. Ces compétences font appel à un réinvestissement de connaissances variées mais de manière simultanée. Nous en avons listé quelques-unes significatives par leurs différences mais suffisamment nombreuses pour éclairer la complexité des difficultés éprouvées par les élèves lors de cet acte : écrire en cursive sur une ligne ou entre deux lignes, maîtriser le sens de l'écriture, le sens du tracé des lettres, avoir conceptualisé les espaces entre les mots, l'organisation spatiale d'une page, la beauté des formes, la tenue correcte de l'outil scripteur, du support, du corps, la coordination motrice des mouvements de poignet et de l'œil qui répète les allers-retours entre le modèle et sa transcription, savoir faire glisser sa main sur le support, comprendre ce que l'on écrit et donc pouvoir se corriger si besoin, comprendre qu'il y aura un lecteur...

\section{L'ÉCRITURE À L'ÉCOLE MATERNELLE, OÙ EN EST-ON ?}

Ajuriaguerra et Auzias (1960) avaient classé les exercices graphiques préparatoires à l'écriture, en deux grandes catégories, au cœur d'une perspective historique de cet enseignement datant du début de l'école maternelle. Le Roux (2004) les rappelle. En premier lieu, les exercices moteurs et sensoriels qui sont travaillés au sein des activités physiques, de l'éducation manuelle et de toutes les activités sensorielles et perceptives. En second, les exercices moteurs qui regroupent des dessins libres ou décoratifs, les tracés libres ou dirigés allant jusqu'à l'étude des lettres. S'il semble difficile de délimiter la fin des exercices préparatoires à l'écriture et le début de l'enseignement de l'écriture proprement dite, il paraît, au regard de ces différentes études, que l'action du sujet produisant une trace et son investissement dans l'acte d'écrire seraient liés au rapport qu'il entretient avec son corps, sa motricité, en dehors de l'acte pur d'écriture : un rapport à l'écriture résolument inséré dans un rapport au monde matériel et social comme le décrit Barré de Miniac (1999).

Pour autant, si l'apprentissage de l'écriture s'enseigne, Auzias (1966, 1977) et Lurçat $(1974,1985)$ insistent sur la nécessité de l'étude de la forme mais aussi de la trajectoire. Tracer dans l'espace, utiliser son corps afin de développer l'action et la perception, favorisent l'appropriation de signes graphiques comme les lettres. 


\section{S. BRIQUET-DUHAZÉ}

L'anticipation des gestes, la nécessaire action intentionnelle du geste sont tout aussi fondamentaux que les aspects formels (Lurçat, 1983).

Zerbato-Poudou $(1998,2000,2004)$ a réalisé une analyse critique des pratiques graphiques liées à l'apprentissage de l'écriture où l'aspect langage écrit semble être très souvent absent. Ces graphismes, éloignés de l'activité d'écriture productive, offrent aux élèves une représentation de la tâche peu propice à l'ancrage d'un savoir, puisqu'il s'agit de répéter des fragments qui devraient permettre aux enfants, en les coordonnant, la reproduction réussie de la graphie d'une lettre.

Ferreiro (1990) rejetait déjà cette attention portée aux aspects formels de l'écriture alors même que son apprentissage ne pourrait avoir lieu qu'avec la compréhension fine du système d'écriture. Son analyse (2000) centrée sur les aspects constructifs, inscrit le développement de l'écriture dans un processus psychogénétique au sens où le système d'écriture étant socialement construit, il devient objet de connaissance et permet, entre autres, l'établissement de liens entre langue orale et les formes graphiques correspondantes. L'interprétation se centre alors sur ce que l'enfant a voulu représenter et comment il y est parvenu. Dans cette perspective, Saada-Robert (2003) et son équipe ont mené des expérimentations de lecture émergente et d'écriture émergente auprès d'enfants de 4 ans, scripteurs sans savoir écrire, montrant ainsi que l'apprentissage de la lecture n'est pas forcément premier comme on le considère très souvent. Zesiger, Deonna et Mayor (2000) situent vers 5-6 ans la période où l'enfant parvient à reproduire les lettres de manière systématique mais avec la persistance de grandes lettres et des irrégularités sur le plan spatial. Cette observation perdure chez les enfants éprouvant de grandes difficultés d'écriture, outre les inversions et les déformations qui ne sont plus présentes chez les premiers.

Plane (2002-2003) présente une synthèse des travaux récents sur l'apprentissage de l'écriture et particulièrement l'ancrage de la didactique dans ce domaine. L'auteur souligne également l'apprentissage collectif de cet acte somme toute très individuel. Ces réflexions reflètent également le caractère polysémique du terme où l'action d'écrire peut tout aussi bien désigner le processus qui se déroule et le réalisé, le produit de ce processus.

\section{QUESTION DE RECHERCHE}

Dès lors, il semblait possible d'envisager l'écriture du prénom comme une activité propice à la conceptualisation de l'écrit, à l'apprentissage scriptural, tant il représente un support affectif important et motivant pour l'enfant. Cependant, Ferreiro (2000) ayant montré qu'il n'y avait pas obligatoirement de relation entre l'écriture du prénom par l'enfant et sa compréhension du code, $\mathrm{j}$ 'ai vérifié que les élèves concernés avaient débuté cette compréhension de manière à pouvoir me concentrer sur l'activité de copie réflexive.

Les élèves de grande section ont beaucoup de difficultés à produire une écriture dont les caractères sont significativement plus petits pour pouvoir entrer dans un cadre formel, celui du double lignage traditionnel des cahiers, notamment. 
À partir du double constat, du travail écrit en grande section, réalisé sur des feuilles de format $\mathrm{A} 4$, et l'usage d'une étiquette prénom dont le modèle varie peu durant la scolarité en cycle 1, en ce qui concerne le format et la hauteur des lettres, on peut se demander si ce sont les dimensions du support d'écrit qui influencent la plus grande taille des lettres produites ou bien, si ce sont plutôt les dimensions de l'étiquette prénom modèle qui induisent la prédominance des grandes lettres. L'expression «taille des lettres » est utilisée ici pour désigner l'écriture manuelle (de l'élève ou de l'enseignant); celle de «police des caractères » a le même sens mais se réfère à une écriture réalisée avec traitement de texte.

Ma question concerne donc l'usage qui est fait, par les élèves de grande section, de l'étiquette prénom et ce qu'il advient lorsque celle-ci varie. En d'autres termes, la diminution de la taille de l'étiquette et des caractères modèles proposés at-elle une incidence sur la taille des caractères reproduits par l'élève ? D'autre part, la réduction du format du support feuille a-t-elle un impact sur la taille des caractères d'autant que cet exercice proposé en fin de grande section relève d'une compétence que l'enfant aura à mettre en œuvre dès le début du C.P. ?

\section{EXPÉRIMENTATION}

Une première observation menée auprès de mes 26 élèves de moyenne et grande sections avait mis en évidence que la taille de l'étiquette prénom avait peu d'incidence sur la diminution des caractères produits par les élèves.

L'une était de format $20 * 12 \mathrm{~cm}$. recto verso :
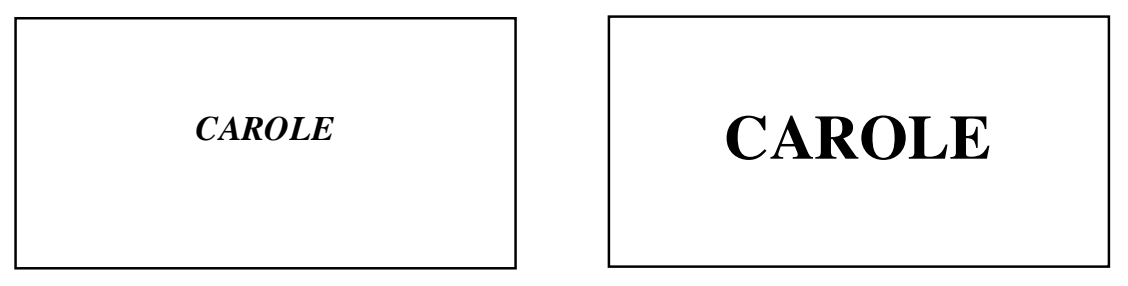

l'autre de $8 * 3 \mathrm{~cm}$. recto verso :

$$
\text { CAROLE }
$$

\section{carole}

Que les élèves choisissent l'une ou l'autre, que ces modèles leur soient présentés successivement, les caractères du prénom écrit par les élèves, demeurent inchangés en taille.

M'interrogeant sur des situations pouvant favoriser la diminution de la taille des caractères, je leur ai proposé de dessiner un bonhomme sur une feuille de format 


\section{S. BRIQUET-DUHAZÉ}

$21 * 29,7 \mathrm{~cm}$ puis de réaliser ce même bonhomme en plus petit sur un format réduit $\left(1 / 8^{\text {ème }}\right.$ de la feuille précédente). En reléguant l'activité d'écriture au second plan, les élèves se sont investis dans le dessin. Je n'ai donné aucune consigne concernant le prénom mais tous savaient qu'il devait y figurer au dos de chaque feuille. Demander aux élèves d'écrire leur prénom en plus petit aurait certainement eu pour conséquence un développement de la peur de l'échec liée à la difficulté presque insurmontable pour certains, que cet exercice représentait. La diminution du format du support et l'activité de dessin impliquaient une écriture plus petite de la taille des lettres. Les résultats obtenus montrent que tous les élèves ont diminué la taille des caractères de leur prénom, qu'il soit écrit, en script ou en cursive. ${ }^{2}$ Certains élèves ont élaboré une réflexion et leur stratégie s'est alors révélée : plusieurs essais afin que toutes les lettres du prénom figurent dans l'espace imparti; changement d'écriture pour certains maîtrisant la cursive mais préférant reprendre l'écriture en capitales d'imprimerie : une réalisation des tracés des lettres plus fine lors de l'écriture sur petit format (Briquet-Duhazé, 2002).

À partir des résultats obtenus précédemment, j'ai réitéré la même investigation l'année suivante auprès d'un échantillon plus large composé de 39 élèves de grande section uniquement. J'ai tout d'abord évalué les aspects figuratifs des productions tels que l'utilisation, la tenue de l'outil scripteur, le tracé conventionnel des lettres, leur orientation, les inversion. J'ai également vérifié la reconnaissance globale de leur prénom ainsi que la construction engagée de la maîtrise du code alphabétique en interrogeant les élèves sur le nom des lettres qu'ils écrivaient, sur les mots écrits en ne privilégiant que l'écriture en majuscules d'imprimerie ainsi que le sens même de l'activité de produire de l'écrit pour être lu.

L'observation individuelle des élèves scripteurs a ensuite été réalisée en situation provoquée et non en situation programmée d'activité de copie à la demande des maîtres titulaires.

La première phase s'est déroulée au cours du second trimestre. J'ai proposé trois étiquettes prénoms de taille et de modélisation variée de manière à ce que la différence marquée la première fois entre une grande et une très petite étiquette, apparaisse moins nettement cette fois et de manière plus progressive. Les formats des étiquettes étaient de $21^{*} 14,5 \mathrm{~cm} ; 14,5 * 10,5 \mathrm{~cm} ; 10,5 * 7,3 \mathrm{~cm}$. Le modèle du prénom de chaque élève figurait en lettres scriptes majuscules de police 20, 36 et 48 soit une hauteur des lettres de 5,8 et $11 \mathrm{~mm}$. Le choix de chaque enfant devait se porter sur l'une d'entre elles (appelées pour et par les enfants, la grande, la moyenne et la petite). La consigne était d'écrire son prénom sans que soit préciser le respect de la taille des lettres. Puis, chacun devait effectuer un second choix avant de prendre la dernière étiquette et d'appliquer la même consigne sur une feuille blanche du format A4.

La seconde phase a eu lieu en juin. Après avoir demandé à chaque enfant s'il se souvenait du travail réalisé quelques mois plus tôt avec les trois étiquettes, je leur

\footnotetext{
${ }^{2}$ Des productions d'élèves sont reproduites dans l'ouvrage suivant: Briquet-Duhazé S. (2005). Différencier sa pédagogie à l'école maternelle. Paris : Bordas.
} 
ai demandé s'ils savaient écrire leur prénom sans l'étiquette modèle (j'avais au préalable, vérifié que cette compétence était acquise auprès des enseignants. Les élèves savaient, par ailleurs, que les étiquettes prénoms étaient à leur disposition s'ils le souhaitaient. Chacun a écrit son prénom sur le même support que la première fois (une feuille blanche de format A4) puis aussitôt sur un support feuille d'un très petit format $(7,5 * 5 \mathrm{~cm})$.

\section{ANALYSE ET INTERPRÉTATION DES RÉSULTATS}

L'analyse des productions des 39 élèves, en janvier, révèle trois groupes distincts. Le premier, appelé groupe A est constitué de 17 enfants dont la particularité est de faire varier la taille des lettres de leur prénom en fonction des trois étiquettes présentées. Le groupe B est composé de 14 élèves qui diminuent la taille des lettres lorsqu'ils utilisent la petite étiquette ; il n'y a aucune différence notable lorsqu'ils prennent la grande et la moyenne. Le groupe $\mathrm{C}$ est formé par 8 autres qui écrivent leur prénom trois fois à l'identique.

Si nous examinons maintenant les productions à l'intérieur de chaque groupe, on observe que :

- Pour le groupe A, non seulement les élèves font varier la taille des lettres en fonction du modèle, mais ils respectent scrupuleusement la police de celui-ci. Leurs trois prénoms sont donc identiques en tout point aux trois étiquettes modèles. De même, l'étude de la plus petite écriture révèle que les caractères (uniquement dans cette police) sont représentés de manière plus fine : le point sur le i fait place au rond, en grands caractères; les accents sont représentés alors qu'ils ne le sont pas avec les caractères plus grands; les lettres sont mieux formées et en particulier mieux fermées (o)...

- Pour le groupe B, l'écriture est souvent plus irrégulière dans le tracé des lettres surtout lorsqu'ils tentent de diminuer les caractères. Quelques-uns font des erreurs dans la copie de leur prénom. La police de caractères qu'ils utilisent pour copier les modèles moyen et grand correspond au modèle moyen. Ces élèves tentent donc de diminuer la taille des lettres de leur prénom.

- Les élèves du groupe $\mathrm{C}$ écrivent souvent très grand et font majoritairement beaucoup de fautes dans la copie du prénom. Lorsqu'ils écrivent avec des caractères qui correspondent au mieux à la police moyenne, les erreurs sont également présentes. Ces enfants ne prennent pas le risque d'écrire plus petit. Trois seulement ont écrit leur prénom en petits caractères, 3 fois à l'identique avec un tracé régulier. Ces derniers n'ont donc pas tenu compte des modèles moyen et grand. Nous considérons donc qu'ils peuvent rejoindre le groupe A puisqu'ils maîtrisent la compétence en jeu.

En juin, lorsque les enfants ont écrit leur prénom sur la feuille blanche A4, après avoir affirmé se souvenir du travail effectué en janvier avec les 3 modèles différents, on constate que : 


\section{S. BRIQUET-DUHAZÉ}

- 16 élèves ont surdimensionné l'écriture du prénom, c'est-à-dire qu'ils ont utilisé une police de caractères supérieure voire très supérieure à celle qui correspondait au grand format. Ce qui est notable, c'est qu'ils appartiennent équitablement aux trois groupes référencés $6 \mathrm{~A}-4 \mathrm{~B}$ et $7 \mathrm{C}$. On peut donc considérer que même ceux qui sont capables de diminuer la taille des lettres de leur prénom en milieu d'année ne poursuivent pas, n'utilisent pas cette compétence au fil du temps, bien au contraire ; et ceux pour qui cela représentait une difficulté, ne se sont pas investis dans cette voie.

- 10 enfants ont écrit leur prénom en utilisant les caractères qui correspondaient à la grande étiquette (2A-2B et $6 \mathrm{C})$.

- 2 ont écrit avec une police correspondant à l'étiquette moyenne (1A-1C)

- 1 seule élève a écrit avec des petits caractères (A).

Aussitôt les élèves ont écrit leur prénom sur le petit format de feuille, blanc également. Aucun n'a échoué à cette commande, seul un élève a eu des difficultés pour écrire la dernière lettre de son prénom par manque de place. 5 ont maintenu la même taille de caractères que sur le grand format car ils écrivaient déjà plus petit : ce sont les trois enfants référencés en dernier et 2 parmi les 19 ayant un prénom comportant peu de lettres. Tous les autres, soit 34 élèves ont diminué la taille des caractères de leur prénom dont, particulièrement, tous ceux qui avaient surdimensionné leur écriture quelques instants auparavant.

En résumé, les points saillants de cette expérimentation sont les suivants :

- les élèves de grande section sont capables de copier leur prénom en réduisant la taille des lettres, en milieu d'année scolaire lorsqu'une étiquette de petit format leur est fournie en même temps que d'autres d'un format supérieur et mieux connu. Notre première observation avait montré que proposer l'étiquette prénom habituellement utilisée par les enfants, en même temps qu'une très petite, n'offre pas un choix suffisamment sécurisant pour que les élèves s'investissent dans la réduction de la taille des caractères.

- en revanche, l'écriture sur le support de petit format avait révélé qu'ils étaient capables de le faire, à la même période de l'année (début du deuxième trimestre). Nous avons obtenu le même résultat avec ce nouvel échantillon. Cependant, le décalage dans le temps (écriture sur un petit support en fin d'année scolaire) mais surtout la comparaison simultanée de cette production avec la même sur un support A4 montrent qu'il n'y a pas de réinvestissement de cette compétence alors qu'elle peut être maîtrisée depuis plusieurs mois, mais surtout qu'il n'y a pas eu d'automaticité permettant un renforcement.

- la diminution du support feuille a plus d'impact sur la diminution des caractères scripturaux produits par les élèves.

- enfin, un modèle sur étiquette présente l'avantage pour l'élève, de pouvoir la positionner à sa convenance. J'ai observé que majoritairement, ils la placent audessus de l'endroit où ils vont produire. Cependant, dès le C.P., les modèles réalisés par les enseignants sur le cahier figurent dans la marge, le plus souvent. 


\section{CONCLUSION}

Outre l'objectif d'étudier une compétence particulière qui est la réduction de la taille des caractères lors de la copie du prénom, cette expérimentation laisse supposer que de jeunes élèves maîtrisent des compétences scripturales en place, non mobilisées car non évaluées et qui feront plus tard, l'objet d'un apprentissage. Révéler à l'enfant la maîtrise d'une compétence et l'entretenir ne peut que l'encourager dans l'investissement de cet apprentissage.

Du point de vue didactique, cela implique que l'enseignant doit veiller à faire varier suffisamment tôt au cours de l'année de grande section, la police de caractères des modèles d'écriture sur les étiquettes proposées ainsi que les formats des supports de manière à ce que d'une part l'élève adapte ses productions scripturales et ait conscience de cette maîtrise de la hauteur des lettres qu'il peut produire ; et d'autre part, que l'enfant puisse poursuivre cette conformité lorsque le format diminue et que le modèle du prénom ne lui est plus utile. On peut supposer que la copie du prénom s'appuyant sur un modèle et un support standards du point de vue du format, peut induire une autre automaticité qui serait pour l'élève, d'écrire son prénom dans une hauteur de caractères sans variabilité. Cet «exercice » répété peut inférer chez certains des difficultés à l'entrée du C.P. En effet, le modèle des cahiers engendre une production scripturale encore plus petite que celle ayant fait l'objet de notre étude et un cadrage entre deux lignes présenté sans détour dans tous les supports formels alors même que les élèves de grande section travaillent le plus souvent sur des supports blancs offrant ainsi une plus grande liberté dans la hauteur des caractères.

Enfin, les recherches dans ce domaine (Lurçat, Ferreiro, Zerbato-Poudou, Saada-Robert...) insistent sur l'aspect social et culturel de l'écriture, la compréhension de sa représentation, le lien nécessaire entre lecture et écriture. Les mêmes auteurs dénoncent la nécessité mais également le danger de la suprématie des aspects formels dans les activités scolaires. L'écriture du prénom en maternelle est l'une des activités privilégiée, sans aucun doute reliée à sa lecture, et favorise l'accès à un niveau de conceptualisation, de représentation, de ce mode de langage tout en travaillant les aspects formels.

Sophie BRIQUET-DUHAZÉ IUFM de Rouen EA CIVIIC Université de Rouen

Abstract : The article raises the question of reduction of the size of letters for fiveyear old children in infant-schools when they write their first name. We suggest the possibility that the model of the name label, as well as the dimensions of the paper used are relevant features when studying the difficulties met by pupils when they have to write between two lines and therefore have to reduce their handwriting when they enter C.P. (first year of primary school, for children aged 6). 


\section{S. BRIQUET-DUHAZÉ}

Key words : infant school, five-year olds, copying one's name, model label, reducing characters, reducing paper used, links between infant and primary school.

\section{Bibliographie}

Ajuriaguerra J. \& Auzias M. (1960) «Méthodes et techniques d'apprentissage de l'écriture » — in : La psychiatrie de l'enfant, III (609-717).

Auzias M. (1966) L'apprentissage de l'écriture. Paris : Bourrelier.

Auzias M. et al. (1977) Écrire à 5 ans ? Paris : PUF.

Barré de Miniac C. (dir.) (1999) Copie et modèle : usages, transmission, appropriation de l'écrit - Actes du Colloque GERI.

Briquet-Duhazé S. (2002) «L'écriture du prénom en maternelle» - La Nouvelle Revue de l'AIS 20 (131-138).

Ferreiro É. (1990) Apprendre le lire-écrire. Lyon : Voies Livres.

Ferreiro É. (2000) L'écriture avant la lettre. Paris : Hachette.

Le Roux Y. (2004) «Comment les enfants apprennent à écrire » - Enfance 24 (8189).

Lurçat L. (1974) Étude de l'acte graphique. Paris, Mouton.

Lurçat L. (1983) «Le graphisme et l'écriture chez l'enfant»-Revue Française de Pédagogie 5 (7-18).

Lurçat L. (1985) L'écriture et le langage écrit de l'enfant. Paris : ESF.

Plane S. (2002-03) «La didactique du français, témoin et acteur de l'évolution du questionnement sur l'écriture et son apprentissage »-Repères 26-27 (3-19).

Saada-Robert M. et al (2003) «Écrire pour lire dès quatre ans, didactique de l'entrée dans l'écrit»-Cahiers de la Section des Sciences de l'Éducation, Genève 100.

Zerbato-Poudou M.T. (1998) «Rôle du contexte dans l'apprentissage premier de l'écriture à l'école maternelle » - Repères 18 (113-122).

Zerbato-Poudou M.T. (2000) «L'apprentissage premier de l'écriture, nouvelles conceptions » - in : R. Amigues et M.T. Zerbato-Poudou (dir.) Comment l'enfant devient élève (133-202). Paris : Retz.

Zerbato-Poudou M.T. (2004) « À quoi servent les exercices graphiques ? »-Rep⿳亠一res 26-27 (61-71).

Zesiger P., Deonna T. \& Mayor C. (2000) «L'acquisition de l'écriture » - Enfance 3 (295-304). 